\title{
Improving the Laboratory Add-On Process and Increasing Housestaff Satisfaction with an EMR Intervention
}

\author{
Vahe Shahnazarian, Parag Mehta \\ New York Methodist Hospital, USA
}

\begin{abstract}
At a community hospital in Brooklyn, New York, the process for ordering add-on testing to drawn blood tubes involved filling out a paper sheet, then faxing and bulleting that sheet to the lab. It was a very inefficient, cumbersome, and unsatisfactory way of completing the process. In light of this, an EMR intervention was implemented in which the add-on order was placed as an EMR order. The study spanned over almost five years, over a year of which was post-intervention. There was a statistically significant increase in the number of add-on orders being placed as a result of the intervention. This has greatly improved housestaff satisfaction with the overall process. In conclusion, the project was a great success and met its goals of simplifying a difficult and cumbersome process while increasing user satisfaction.
\end{abstract}

\section{Problem}

At New York Methodist Hospital, a 651-bed community hospital in Brooklyn, New York, if a physician wanted to order an add-on to drawn labs, it was a very cumbersome process. If the appropriate type of blood tube was in the lab, the physician would fill out a "laboratory add-on sheet," select the appropriate lab tests, and fax the sheet to the lab. Then, they would send the sheet to the lab using the pneumatic bullet system, call the lab to confirm receipt and wait for the test to be done. This sheet would be handwritten, which meant the handwriting needed to be pristine in order to avoid miscommunication. The correct patient information would have to be entered on the sheet as well. It was difficult finding the sheets on the floor and locating a fax machine. Also, one would have to find the fax number, then locate a pneumatic bullet tube. Once it was received by the lab, the physician would call the lab, wait on hold while the technician found the faxed and bulleted sheet, then confirm everything over the phone. It was very labor intensive, time consuming, and inefficient. Inevitably, this led to many providers foregoing the process and re-ordering STAT labs, which resulted in patients having more phlebotomy done. This caused the patient discomfort; it was also a waste of blood and resources.

\section{Background}

Currently in the Western world, even with new laboratory technology, approximately 25 million liters of blood are discarded due to drawing too much blood into the tube. This totals more than four times the volume of blood transfusions in one year.[1] In fact, studies have shown the phlebotomist or nurse draws more blood into the tube than is required. One study showed a mean of 45 times the amount of blood that was needed was being drawn into the tubes. This led to 200 to $550 \mathrm{~mL}$ of extra blood being drawn from the patient during their hospitalization.[2] Blood transfusions are being closely scrutinized as of late in order to avoid unnecessary transfusions. Diagnostic phlebotomy may be significantly contributing to iatrogenic anemia, especially in the critically ill. In the intensive care unit, $2-10 \mathrm{~mL}$ of blood may be withdrawn from the catheter as "waste" prior to the actual sample being drawn (which itself may be $2 \mathrm{~mL}$ or more).[3] Even with interventions in place to help avoid needle stick injuries, they still occur. With them comes the significant psychosocial burden of worrying about infectious disease as well as the increased cost of testing and treating the injured provider.[4]

\section{Baseline measurement}

From 2010 to 2013 , every inpatient was included in this study. For this study, the total number of laboratory tests ordered and the total number of laboratory add-on tests ordered were quantified. These two values were chosen in order to show the ratio of lab add-ons to regular lab orders. A total of 5,845,983 laboratory tests were ordered on those patients. Of those, 89,818 were add-on tests, ordered using the process described above. Only $1.54 \%$ of the total lab tests ordered used the add-on system. Also, focus groups were created using randomly selected housestaff physicians in order to examine user satisfaction. They were verbally surveyed regarding their satisfaction with the current process for ordering add-on labs. The overwhelming consensus was that no one was satisfied with the current procedure and a change was needed to streamline the process. The full data set can be viewed in the supplemental section. These three measures were chosen because they would allow for a demonstration of either an increase or decrease in the number of lab add-ons orders, the percentage of lab add-ons to regularly ordered labs, and the change in user satisfaction. This would show that the new process had either been embraced or rejected by the housestaff, which would in turn show if more or less add-ons were ordered with a simpler and more efficient EMR order.

See supplementary file: ds7452.pdf - "Full Data Set in Tables \& Graphs"

\section{Design}


The aim of this project was to show that there would be an increase in the number of laboratory add-ons when using a simplified EMR order. In close conjunction with the Information Technology Services Department (IT), it was decided a new Electronic Medical Record ordering system would be the most effective form of addressing the problem. An order would be added to the EMR titled "Lab Add-On." Once this order was clicked, the provider would manually type any requested lab studies into the appropriate field. It could be requested as routine, urgent, or stat. A pager number would be required in case there was a problem fulfilling the order. Once the order was signed, it would print the requisition in the lab. A technician in the lab would take the requisition and add the requested test. The order would then populate into the EMR, signaling the provider that the study was underway, and then the result would appear in the EMR upon completion. This new process was implemented in 2014

\section{Strategy}

The Plan, Do, Study, Act (PDSA) model was used for this quality improvement study. This model uses four distinct phases. In the Plan phase, the objectives are listed and a plan to address them is formulated. In the Do phase, the plan is implemented and detailed data are collected prior, during, and after the implementation. In the Study phase, all the collected data are analyzed. Finally, in the Act phase, the intervention is studied and the decision is made to adopt, adapt, or abandon it depending on the gathered data. The cycle is then repeated for all further adaptations of the intervention. The total number of cycles depends on the amount of change, if at all, that is accomplished by the intervention. $[5,6]$

\section{PDSA Cycle 1}

In the first cycle, the aim was to develop an EMR intervention for a lab add-on process and to measure the change in amount of lab add-ons that were ordered, as well as the user satisfaction associated with the intervention. It was thought that the number of add-on orders would increase and that the user satisfaction would also increase. The new EMR order was implemented in January 2014. An immediate increase in the percentage of lab add-on orders to regularly ordered labs was noted. Also, the focus groups were reconvened and expressed an increase in their satisfaction with the new process. However, a few problems were noted. First, the laboratory technicians were becoming overwhelmed by the additional number of lab add-on orders. Second, the printer in the laboratory would occasionally malfunction, which would result in the add-on order being lost and not completed. An action plan was implemented where an extra laboratory technician was hired to cope with the additional volume of tests. Also, the IT Department intervened and fixed the printer issue on their end, which resolved the problem of the lost orders.

PDSA Cycle 2

In the second cycle, the aim was to fine-tune the new order process. This second cycle was studied from February 2014 until October 2015. Again, the change in lab add-on orders and user satisfaction would be measured. There was a continued increase in the percentage of laboratory add-on tests to regularly ordered tests. This time, there were no further complaints from the focus groups. Once the printer issue had been resolved, there had been no further missed lab add-on orders. Also, the laboratory was pleased with the addition of the new technician and they were able to keep up with the increased workload. Even though there were more lab tests being ordered via the add-on system, the technician was able to keep up with the inputting and there was no problem for the lab machinery to cope with the higher volume of tests. With the increase in the number of lab add-ons that were being ordered, the antiquated paper sheets were no longer needed. This eliminated all handwriting, faxing, and bulleting issues from the past.

\section{Results}

Once the intervention was implemented in 2014, it was studied until October of 2015. It was noted that 2,943,530 laboratory tests were ordered, of which 107,427 were add-on orders. This was in contrast to 89,818 add-on tests ordered in the pre-intervention measurement. This was a significantly large increase ( $p$-value of 0.001 ), especially given the fact that the pre-intervention time span was three years while the post-intervention was less than two years. The percentage of lab add-ons to regular orders increased from $1.54 \%$ to $3.65 \%$ during the intervention. Again, focus groups of the same housestaff as before were verbally surveyed. The overall consensus was increased satisfaction with the new process. It was far easier and more efficient than the prior process. The only complaints stemmed from when the printer would malfunction, but that had been resolved by the end of the first PDSA cycle. The full data set can be viewed in the supplemental section.

See supplementary file: ds7453.pdf - "Full Data Set in Tables \& Graphs"

\section{Lessons and limitations}

The 107,427 lab add-ons ordered in a 22 month period during the intervention (as opposed to 89,818 in a 48 month pre-intervention period) represented a large increase from the baseline. The more efficient, simpler process prompted providers to use the add-on option as opposed to just re-ordering STAT labs. In turn, this increased housestaff satisfaction.

There were a number of limitations that were noted once the project was completed. There was no feasible method of measuring costsavings related to the increase in add-ons. The number of complications from the (theoretically) decreased number of additional sticks were also not measured. Due to the enormity of the project, it was also not practical to measure patient satisfaction in an objective manner (survey or otherwise). Also, while housestaff satisfaction was measured, it was done so informally. A more formal and objective method would have been preferred.

\section{Conclusion}

Given the low satisfaction and inefficiency of the initial add-on process, an intervention was implemented using the hospital EMR. 


\section{BMJ Quality Improvement Reports}

The number of lab add-on tests significantly increased when using the new and simple, yet efficient ordering process. Housestaff satisfaction was overwhelmingly increased with this intervention as well. The project was a great success and met its goals of simplifying a difficult and cumbersome process while increasing user satisfaction. Also, this project is completely sustainable and generalizable given it's insertion into the EMR. Any hospital with an EMR can replicate it, and unless it is purposely removed from the EMR, it will remain within the order set permanently. Further steps would be to perform a more formal survey of randomly selected housestaff and patients in order to better demonstrate the effect this has had on them. It would also be prudent to measure the amount of STAT labs still being done in order to see if further improvements can be made to the add-on system in order to reduce the number of STAT labs.

This study highlights the importance of housestaff driven change. Using the EMR, a positive change was accomplished in the daily workflow as well as increasing overall satisfaction. Housestaff can make a significant difference in their environment. Raising awareness of this fact will not only empower more providers to take initiative but will also drive a culture of proactive involvement and quality improvement.[6,7]

\section{References}

1 Levi M. Twenty-five million liters of blood into the sewer. J Thromb Haemost 2014; 12: 1592.

2 Dale JC \& Pruett SK. Phlebotomy - A Minimalist Approach. Mayo Clin Proc 1993; 68: 249-255.

3 Fowler, RA \& Berenson M. Blood conservation in the intensive care unit. Crit Care Medicine 2003; 31: 715-720.

4 McCormick RD, Meisch MG, Ircink FG, et al. Epidemiology of hospital sharps injuries: a 14-year prospective study in the preAIDS and AIDS era. The American Journal of Medicine 1991; 91 : 301-307

5 Taylor MJ, McNicholas C, Nicolay C, et al. Systematic review of the application of the plan-do-study-act method to improve quality in healthcare. BMJ Qual Saf 2013 10.1136/bmjqs-2013-001862

6 Schriefer J, Leonard MS. Patient Safety and Quality Improvement: An Overview of QI. Pediatr Rev 2012;33:353-60.

7 Jones AC, Shipman SA, Ogrinc G. Key characteristics of successful quality improvement curricula in physician education: a realist review. BMJ Qual Saf 2015;24:77-88.

\section{Declaration of interests}

There are no interests to declare related to this research study.
Mr. Jordan Martens and the rest of the IT Services staff, for their hard work and dedication to improving the EMR and workflow.

\section{Ethical approval}

No ethical approval was required for this project. It did not involve any intervention on human subjects. This was an Institutional Review Board (IRB) approved study.

\section{Acknowledgements}

